\title{
Modeling of fatigue behavior based on interaction between time- and cyclic-dependent mechanical properties
}

\author{
A. Vahid Movahedi-Rad, Thomas Keller, Anastasios P. Vassilopoulos ${ }^{1}$ \\ Composite Construction Laboratory (CCLab), \\ Ecole Polytechnique Fédérale de Lausanne (EPFL), \\ Station 16, Bâtiment BP, CH-1015 Lausanne, Switzerland
}

\begin{abstract}
:
A general constitutive equation was established using the theory of viscoelasticity in order to consider the interaction of the time- and cyclic-dependent mechanical properties of laminated composites. This equation was solved for two specific loading patterns, 1) stress unloading to zero stress level (recovery solution), and 2) load control sinusoidal loading (fatigue solution), and was subsequently imported to model tensile-tensile interrupted fatigue experiments (including recovery phases) of $\pm 45^{\circ}$ angle-ply glass/epoxy composite laminates at different stress levels. The viscoelastic parameters in the recovery solution were calibrated at different percentages of fatigue lifetime using the experimental recovery results. The estimated viscoelastic parameters were then imported into the fatigue solution to predict the fatigue stiffness, hysteresis loop area, cyclic creep, storage and loss moduli as well as $\tan (\delta)$ under cyclic loading. The theoretical predictions compared well to the experimental data.
\end{abstract}

Keywords: Fatigue; Recovery; Modeling; Viscoelasticity; Interrupted loading

\footnotetext{
${ }^{1}$ Corresponding author: e-mail: anastasios.vasilopoulos@epfl.ch, Tel: +41216936393; Fax: +41216936240.
} 


\section{Introduction}

Fiber-reinforced polymer (FRP) composites are used in a wide range of applications such as wind turbine rotor blades, airplanes, and bridge decks and are subjected to different types of loading patterns during operation. The behavior of these materials is sensitive to the loading pattern due to their cyclicand time-dependent mechanical properties [1,2]. The cyclic-dependent mechanical properties, such as fatigue stiffness and hysteresis loop area, are mainly linked to high frequency fatigue experiments at zero mean stress level in which the time-dependent deformation of the material does not have a significant effect. On the other hand, the time-dependent mechanical properties are related to the rheology of the material when it is subjected to a sustained load $[2,3,4]$.

Constitutive equations to describe the time-dependent mechanical properties were established based on either physical or phenomenological approaches. Following the physical-based approach, the material behavior is studied on the microscopic scale in order to model, for instance, the uniaxial loading behavior of polymer-based materials including yielding, work-hardening, strain rate and temperature effects $[5,6]$. Hysteresis loops resulting from quasi-static uniaxial tensile loading-unloading cycles of viscoelastic materials were also simulated using a physical-based approach $[7,8,9,10,11,12,13,14]$. This was achieved by modeling the specimen's nonlinear viscoelastic behavior during the loading and unloading phases where the challenge was to model the non-linear unloading phase since the material behaved differently during loading and unloading. To achieve this, new models, based on different approaches, were developed. For example, Colak [13] developed a model to describe the nonlinear ratedependent loading and nonlinear unloading behavior by introducing a variable into the elastic strain rate relationship. Krasnobrizha et al [14] developed a model to describe the evolution of the hysteresis behavior during loading and unloading of laminated composites taking into account the viscoelastoplastic behavior of damaged material.

Phenomenological approaches, studying the material on the macroscale, e.g. [15,16,17], were successfully applied to model the laminated composites' creep, recovery, and relaxation in the linear viscoelastic domain amongst other things. Of the different techniques, the Boltzmann superposition formulation is the most commonly used to model the time-dependent behavior of laminated composites in the linear domain $[18,19,20]$. Although linear viscoelastic principles were useful for the description of the long-term properties, they were inadequate in cases where the stress levels applied were so high that non-linear creep was observed [15,20,21]. Efforts for modeling the non-linear creep behavior of polymers have concentrated on the modification of the Boltzmann superposition formulation. Schapery [22] modified the Boltzmann superposition formulation by adding four time-independent but stress- 
dependent parameters, which expressed the degree of non-linearity in elastic, viscoelastic and permanent strains.

Most constitutive equations used to model cyclic-dependent material behavior were based on phenomenological approaches [23,24,25,26,27,28,29,30,31]. Such models describe fatigue damage by using an evolutionary law to simulate the gradual degradation of the specimen's properties. Damage mechanisms are not considered in these methods, although appropriate damage metrics (such as fatigue stiffness, fatigue strengths, residual strength etc.) are used for the assessment of damage. These damage metrics depend on many factors, including the applied cyclic stress, number of fatigue cycles, loading frequency and environmental conditions [27].

Most of the models in the literature describe the time- and cyclic-dependent mechanical properties separately. However, the time- and cyclic-dependent mechanical properties of laminated composites usually interact with each other, and the degree of interaction depends on the loading spectrum and the material configuration $[32,33,34,35,36]$. In continuous fatigue, the interaction between the time- and cyclic-dependent mechanical properties was studied by monitoring the evolution of fatigue hysteresis loops $[33,34,36,37]$ attributing the shift of the fatigue hysteresis loops to creep effects and the slope of the loops to degradation of the fatigue stiffness due to damage [36]. The interaction between the timeand cyclic-dependent mechanical properties was also studied by applying more complicated loading patterns in which continuous-fatigue was interrupted in different ways $[1,2,34,38]$. Interruption of cyclic loading could also change the fatigue life depending on the applied stress level and material type, as presented in [1,2]. Vieille et al [34] showed that fatigue life could be extended with prior creep in an angle-ply carbon/PPS thermoplastic composite depending on the loading conditions at temperatures higher than $T_{g}$. Similarly, in an angle-ply thermoset graphite/epoxy composite, it was shown that sustained periods of static loads have significant retardation effects on damage propagation and extended fatigue life [38]. Recently, it was shown that the effect of creep on the fatigue life of angle-ply thermoset laminates could be positive or negative deepening on the applied stress level and the hold time [2].

According to the aforementioned studies, the time- and cyclic-dependent properties of composite systems affect each other when the mean stress is not zero and therefore both should be considered in the constitutive equation for an appropriate description of the fatigue behavior. Previous works described in the literature modeled the hysteresis loops during quasi-static tensile loading-unloading; however, the modeling of hysteresis loop behavior (including their shifting as a result of creep) during the fatigue loading has not yet been performed. To the authors' best knowledge, models developed to link the time- and cyclic-dependent mechanical properties mainly focused on creep effects in continuous 
fatigue to improve fatigue life predictions, e.g. in [32,20]. However, a general model to simulate the fatigue behavior (the evolution of fatigue stiffness, hysteresis loops, cyclic creep, $\operatorname{Tan}(\delta)$, storage modulus, and loss modulus) of composite materials has not yet been developed.

The objective of the present work is to derive a general constitutive equation to link and simulate the time- and cyclic-dependent mechanical properties of viscoelastic materials. For this purpose, a general constitutive equation was developed and solved for two specific loading patterns of 1) an unloading to zero stress level (recovery solution) and 2) load control cyclic loading (fatigue solution). To implement this equation, a loading pattern comprising a constant amplitude cyclic loading with repetitive interruptions at zero stress level was selected and applied on matrix-dominated $\pm 45^{\circ}$ angle-ply glass/epoxy composite laminates in which the time-dependent effect was significant. The viscoelastic parameters were estimated by fitting the recovery solution to the experimental recovery curves. The obtained viscoelastic parameters were imported into the fatigue solution to predict the fatigue behavior at different numbers of cycles and stress levels. The accuracy of prediction was evaluated by comparing the results with those obtained from relevant experiments performed in a previous work of the authors.

\section{General constitutive equation}

To model the behavior of a viscoelastic material, different arrangements of springs and dashpots can be used depending on the material behavior [7,10,11,44]. A linear spring with the elastic modulus $E$ simulates the instantaneous elastic deformation (Eq. 1) while a linear dashpot element simulates the viscous deformation (Eq. 2) in which the constant $\eta$ is the viscosity coefficient.

$\sigma=E \varepsilon$

$\sigma=\eta \frac{d \varepsilon}{d t}$

The Maxwell unit is a two-element model consisting of a linear spring element and a linear viscous dashpot element connected in series (Eq. 3) while the Kelvin unit is when the spring element and dashpot element are connected in parallel (Eq. 4).

$\varepsilon(t)=\left(\frac{1}{E}+\frac{1}{\eta \frac{\partial}{\partial t}}\right) \sigma(t)$

$\varepsilon(t)=\left(\frac{1}{E_{1}+\eta_{1} \frac{\partial}{\partial t}}\right) \sigma(t)$

In order to simulate the viscoelastic response of complex material systems under different loading patterns such as creep, relaxation, recovery or cyclic loading, combinations of springs and dashpots in series and/or in parallel configurations can be used. A generally assumed form of a system of springs 
and dashpots is shown in Fig. 1. An independent spring is used to model the elastic strain, while Kelvin units are used to model the viscoelastic strain, and an independent dashpot is used to model the remaining strain after recovery. The corresponding general constitutive equation is shown below.

$$
\varepsilon(t)=\left(\frac{1}{E}+\frac{1}{E_{1}+\eta_{1} \frac{\partial}{\partial t}}+\frac{1}{E_{2}+\eta_{2} \frac{\partial}{\partial t}}+\cdots+\frac{1}{E_{i}+\eta_{i} \frac{\partial}{\partial t}}+\frac{1}{\eta \frac{\partial}{\partial t}}\right) \sigma(t)
$$

This general constitutive equation can be solved for different loading patterns, e.g. 1) for stress unloading from a mean to a zero stress level (a recovery solution), and 2) for load-control sinusoidal cyclic loading (a fatigue solution), as demonstrated using interrupted fatigue experiments, which comprised phases of cyclic loading and repetitive loading interruptions. The specimen recovery behavior during the loading interruptions was modeled using the recovery solution, and correspondingly, the evolution of the viscoelastic parameters was quantified as the number of loading blocks increased. The fatigue behavior at different numbers of cycles was then predicted by importing the estimated viscoelastic parameters into the fatigue solution. The flowchart of the different modeling stages is shown in Fig. 2.

\section{Recovery solution and application}

\subsection{Experimental investigation and recovery results}

Interrupted fatigue tensile experiments were performed on rectangular fully-cured glass/epoxy $[ \pm 45]_{2 \mathrm{~s}}$ specimens with dimensions of $250 \times 25 \times 2.3 \mathrm{~mm}^{3}$ (length $\times$ width $\times$ thickness) and a fiber content of $62 \%$ by volume. Fig. 3 shows a schematic representation of the applied loading profile, comprising repetitive loading blocks of constant amplitude interrupted by unloading periods up to specimen failure. For each experiment, the load was increased until the mean value was reached, after $60 \mathrm{~s}$. Subsequently, the constant amplitude fatigue was applied for a predetermined number of cycles $(20 \%$ of the average continuous fatigue life), followed by a zero-load interval lasting two hours. The cyclic loading was performed in the range of maximum stress levels of $47 \mathrm{MPa}-68 \mathrm{MPa}$. The stress ratio, $R=\sigma_{\min } / \sigma_{\max }$, was kept constant at 0.1 and a constant loading rate of $30.5 \mathrm{kN} / \mathrm{s}$ was used; the frequencies thus varied. The details of the fabrication process, experimental set-up and instrumentation are given in [1].

Figure 4 shows the strain response at the end of a cyclic loading and after the load removal of a selected specimen during one of the loading interruptions. While the cyclic stress instantly decreased to zero, the strain showed a gradual decrease, i.e. first a very sudden elastic strain reduction at the moment of unloading, followed by a viscoelastic strain reduction down to a remaining unrecovered strain after $2 \mathrm{~h}$. It is well documented that specimen recovery, as a result of the viscoelastic nature of the polymeric 
matrix, is responsible for the observed time-dependent decrease of strain [20]. It was shown that the viscoelastic recovery could be described as a three-stage time-dependent strain reduction. Stage I represented a rapid specimen viscoelastic recovery after the elastic strain reduction, and Stage III illustrated the long-term recovery behavior of the specimen in which the strain changed very slowly. Between these two stages, there was a transition region (Stage II), which linked the former and the latter. The remaining strain after a 2-h recovery was attributed to the unrecovered viscoelastic strain and also the accumulated permanent strain due to the fatigue damage.

\subsection{Recovery solution}

The elastic and remaining strains can be calculated by Eq. 6, based on the Maxwell unit, and the viscoelastic strain can be calculated by the Kelvin units, Eq. $7[20,45]$.

$\varepsilon_{\text {Maxwell }}(t)=\frac{\sigma_{m}}{E}+\frac{\sigma_{m}}{\eta}\left(t_{1}-t_{0}\right)$

$\varepsilon_{\text {Kelvin }}(t)=\frac{\sigma_{m}}{E_{i}}\left(1-e^{-\lambda_{i}\left(t-t_{1}\right)}\right)$

where the mean stress is symbolized with $\sigma_{m}$, and $\lambda$ is equal to $E / \eta$ in the Kelvin unit, $t_{0}$ is the point at which the specimen was subjected to the cyclic loading, and $t_{1}$ is the stress removal time. The total strain after the load removal is the sum of all the aforementioned strain components of elastic strain, three stages of viscoelastic strain, and remaining strain (see Fig. 4) and is calculated by Eq. 8:

$$
\begin{aligned}
& \varepsilon(t)=\frac{\sigma_{m}}{E}+\frac{\sigma_{m}}{E_{1}}\left(1-e^{-\lambda_{1}\left(t-t_{1}\right)}\right)+\frac{\sigma_{m}}{E_{2}}\left(1-e^{-\lambda_{2}\left(t-t_{1}\right)}\right)+\frac{\sigma_{m}}{E_{3}}\left(1-e^{-\lambda_{3}\left(t-t_{1}\right)}\right)+\frac{\sigma_{m}}{\eta}\left(t_{1}-t_{0}\right) \\
& \checkmark \sim \\
& \text { Elastic } \\
& \text { Time-dependent } \\
& \text { recovery, Stage I } \\
& \text { Time-dependent } \\
& \text { recovery, Stage II } \\
& \text { Time-dependent } \\
& \text { recovery, Stage III } \\
& \text { Remaining } \\
& \text { Viscoelastic strain }
\end{aligned}
$$

The viscoelastic parameters related to the independent spring and dashpot elements $(E$ and $\eta)$ are estimated directly by measuring the elastic and remaining strains. The parameters of the viscoelastic terms of Eq. 8 are calibrated using the experimental data in the three regions of the viscoelastic part of the recovery curves. The corroboration process was carried out using the Orthogonal Distance Regression (ODR) iteration algorithm [46], and a Chi-Square tolerance value of $10^{-9}$ was reached in all cases. 


\subsection{Estimation of viscoelastic parameters}

Figures $5 \mathrm{a}$ and $5 \mathrm{~b}$ show the recovery curves as the number of loading blocks increased at stress levels of $58 \mathrm{MPa}$ and $49 \mathrm{MPa}$, respectively. The recovery behavior was simulated using Eq. 8, as described above. The obtained recovery curves closely followed the experimental curves. The resulting viscoelastic parameters related to the elastic, viscoelastic, and remaining strains and those of three more stress levels are shown in Fig. 6. Each recovery curve is represented by one data point. The behavior of each viscoelastic parameter (VP) could be simulated by power law equations of the type $V P=\alpha N^{\beta}$ in which $\alpha$ and $\beta$ are fitting parameters. All parameters, except $\eta$, gradually decreased with the number of cycles. An initial steep decrease during the first $10-15 \%$ of the lifetime was followed by a steady-state decreasing trend. Furthermore, with increasing stress levels, the values of the parameters generally decreased, except for $\mathrm{E}_{2}$ and $\eta_{2}$, where no clear trend could be recognized, which was attributed to the fact that the second stage of recovery was a transition stage from short- to long-term recovery. The decreasing trends of the viscoelastic parameters were attributed to the fatigue damage accumulation as shown in [1]. In addition, according to [2], creep at these stress levels was non-linear, which contributed to the change in the viscoelastic parameters. Unlike the other parameters, the evolution of $\eta$, attributed to the remaining strain, showed a growing trend, which was attributed to the decreasing rate of remaining strain (Eq. 8), which subsequently became constant as the number of blocks increased.

\section{Fatigue solution, application and validation}

\subsection{Fatigue solution}

The sinusoidal cyclic stress with a stress amplitude, $\sigma_{a}$, and a given fatigue frequency, $f$, was applied according to the following relationship:

$\sigma(t)=\sigma_{m}+\sigma_{a} \sin (\omega t)$

where $\omega$ is equal to $2 \pi f$. By substituting the cyclic stress equation (Eq. 9) in the general constitutive equation (Eq. 5), the fatigue strain can be calculated as follows (see Annex I):

$\varepsilon(t)=\varepsilon_{c}(t)+\varepsilon_{a} \sin (\omega t-\delta)$

where $\varepsilon_{c}(t)$ is the average cyclic strain, $\varepsilon_{a}(t)$ the strain amplitude, and $\delta$ the phase-lag. The strain calculated by Eq. 10, which is similar to the equation of applied stress (Eq. 9) except that the phase-lag, $\delta$, appears in the sinus term. The terms introduced in Eq. 9 and Eq.10 are schematically shown in Fig. 7. The corresponding equations for the calculation of $\varepsilon_{c}(t), \varepsilon_{a}(t)$, and $\delta$ are shown in Eqs. 11-13, respectively (see Annex I). 


$$
\begin{aligned}
& \varepsilon_{c}(t)=\frac{\sigma_{m}}{E}+\sum_{i=1}^{i=3} \frac{\sigma_{m}}{E_{i}}\left(1-e^{-\lambda_{i}\left(t-t_{0}\right)}\right)+\frac{\sigma_{m}}{\eta}\left(t-t_{0}\right) \\
& \begin{array}{c}
\begin{array}{c}
\text { Elastic } \\
\text { strain }
\end{array} \\
\text { Viscoelastic strain }
\end{array} \\
& \varepsilon_{a}=\sigma_{a} \sqrt{\left(\sum_{i=1}^{i=3} \frac{1}{\eta_{i}} \frac{\lambda_{i}}{\lambda_{i}^{2}+\omega^{2}}+\frac{1}{E}\right)^{2}+\left(\sum_{i=1}^{i=3} \frac{1}{\eta_{i}} \frac{\omega}{\lambda_{i}^{2}+\omega^{2}}+\frac{1}{\eta \omega}\right)^{2}} \\
& \delta=\tan ^{-1}\left(\frac{\sum_{i=1}^{i=3} \frac{\omega}{\eta_{i}\left(\lambda_{i}^{2}+\omega^{2}\right)}+\frac{1}{\eta \omega}}{\sum_{i=1}^{i=3} \frac{\lambda_{i}}{\eta_{i}\left(\lambda_{i}^{2}+\omega^{2}\right)}+\frac{1}{E}}\right)
\end{aligned}
$$

\subsection{Validation of implemented solutions}

In each experiment, the fatigue strain was simulated by importing the estimated viscoelastic parameters into Eqs. 11-13 to calculate the cyclic creep strain, cyclic strain amplitude, and phase-lag, respectively. The hysteresis loops were simulated by plotting the applied cyclic stress (Eq. 9) against the fatigue strain (Eq. 10) for each cycle. The detailed evaluation of the obtained results was carried out by comparing the hysteresis loop area, fatigue stiffness, and average cyclic strain with the experimental results obtained from the cyclic phase of the loading pattern (see Fig. 3) at the different numbers of cycles and stress levels.

The experimental fatigue hysteresis loops at $64 \mathrm{MPa}$ and $49 \mathrm{MPa}$ are indicated by dots in Fig. 8a and $9 \mathrm{~b}$, respectively. The hysteresis loop area, fatigue stiffness, and average cyclic strain exhibited measureable changes as the number of fatigue cycles increased for both high and low cyclic stresses. The predicted hysteresis loops are also indicated in Fig. $8 \mathrm{a}$ and $8 \mathrm{~b}$ by solid lines. The experimental and predicted hysteresis loop areas, fatigue stiffnesses and average cyclic strains are compared in detail in Figs. 9-11.

The variations of the experimental and predicted hysteresis loop areas per cycle versus the normalized number of cycles are shown in Fig. 9 at high and low stress levels. The hysteresis area increased gradually with the number of cycles. At the high stress level, the experimental and predicted curves compared well. At the low stress level, the predicted curve however slightly underestimated the experimental results, which showed that the fatigue solution using the estimated viscoelastic parameters from the recovery curve could not fully simulate the hysteresis loop area.

The variation of the experimental and predicted fatigue stiffness versus the normalized fatigue life is shown in Fig. 10 for high and low stress levels. In both cases, an initial steep decrease during the first $10-15 \%$ of the lifetime, followed by a steady-state stiffness decrease up to specimen failure, was 
observed. Experimental and predicted results compared well at both stress levels, with the exception of the initial stage at the high stress level, where the former were slightly overestimated.

The variation of the average cyclic strain (average of maximum and minimum strains in one cycle) versus the normalized number of cycles is shown in Fig. 11. Independent of the stress level, the strains showed an increasing trend in the form of a ternary curve. The first region was at the early stage of cyclic loading in which the average cyclic creep strain rapidly increased, however at a decreasing rate. During the second stage, the average cyclic strain increased at a steady rate, while, finally, a rapid increase was observed prior to failure. At the beginning of each loading block, a gradual increasing of the cyclic strain was observed due to specimen recovery during the cyclic stress interruption at zero stress level [1]. The predicted average cyclic strains versus number of cycles are also indicated in Fig. 11 by green curves. The fatigue solution predicted well the general trend of the average cyclic strain as well as the initial increase in each loading block at both stress levels.

In addition to the average cyclic strain, the evolution of the strain components, i.e., the elastic, viscoelastic, and remaining strains were also obtained and are shown in Fig. 11. As the number of cycles increased, all strain components increased as a result of the fatigue damage formation and accumulation. The rate of increase of the different strain components varied between the two stress levels. The remaining strain was observed to increase more rapidly than the elastic and viscoelastic strain components and was the main reason for the growth of the cyclic strain. Additionally, viscoelastic strains were composed of small segments attributed to each loading block; they exhibited an initial increase and then a steady-state stage.

The storage and loss moduli of viscoelastic materials measure the stored energy, representing the elastic portion, and the dissipated energy, representing the viscous portion [47]. The derived constitutive equation is also capable of simulating the evolution of the storage and loss moduli and $\tan (\delta)$ with the number of cycles.

$\tan (\delta)$ derived from experiments at each cycle was calculated using the following equation, as derived in [48]:

$\sin (\delta)=\frac{E w}{\pi \sigma_{a}^{2}}$

where $w$ is the hysteresis loop area of cycle $N . E$ and $w$ were obtained from the experiments at the applied stress amplitude $\sigma_{a}$. The magnitude of the storage and loss moduli in each cycle was then determined by solving the two following equations: 
$\tan (\delta)=\frac{E^{\prime \prime}}{E^{\prime}}$

$E=\sqrt{{E^{\prime 2}}^{2}+E^{\prime \prime 2}}$

where $E^{\prime}$ and $E^{\prime \prime}$ are the storage modulus and loss modulus, respectively. The evolution of the resulting experimental storage modulus, loss modulus, and $\tan (\delta)$ are shown in Fig. 12 for high and low stress levels, respectively. Accordingly, as the number of cycles increased, the storage modulus decreased, whereas loss modulus and $\tan (\delta)$ gradually increased. As shown in [1], damage gradually formed and propagated as the number of cycles increased, which reduced the storage modulus, while it also caused more dissipation of energy due to the internal friction that augmented the magnitudes of the loss modulus and $\tan (\delta)$.

Based on the evolution of the viscoelastic parameters estimated at different numbers of cycles (Fig. 6), the storage and loss moduli as well as $\tan (\delta)$ were simulated according to Eq. 17, Eq. 18, and Eq. 13 respectively. The mathematical derivation of these equations is given in Annex II. The comparison of the predicted and experimentally derived storage modulus showed good agreement; however, the loss modulus and $\tan (\delta)$ were generally slightly underestimated. The lack of accuracy in predicting the loss modulus and $\tan (\delta)$ confirmed that the fatigue solution could not fully simulate the amount of energy dissipated at low stress levels.

$$
\begin{aligned}
& E^{\prime}=\frac{\sum_{i=1}^{i=3} \frac{\lambda_{i}}{\eta_{i}\left(\lambda_{i}^{2}+\omega^{2}\right)}+\frac{1}{E}}{\left(\sum_{i=1}^{i=3} \frac{\lambda_{i}}{\eta_{i}\left(\lambda_{i}^{2}+\omega^{2}\right)}+\frac{1}{E}\right)^{2}+\left(\sum_{i=1}^{i=3} \frac{\omega}{\eta_{i}\left(\lambda_{i}^{2}+\omega^{2}\right)}+\frac{1}{\eta \omega}\right)^{2}} \\
& E^{\prime \prime}=\frac{\sum_{i=3}^{i=3} \frac{\omega}{\eta_{i}\left(\lambda_{i}^{2}+\omega^{2}\right)}+\frac{1}{\eta \omega}}{\left(\sum_{i=1}^{i=3} \frac{\lambda_{i}}{\eta_{i}\left(\lambda_{i}^{2}+\omega^{2}\right)}+\frac{1}{E}\right)^{2}+\left(\sum_{i=1}^{i=3} \frac{\omega}{\eta_{i}\left(\lambda_{i}^{2}+\omega^{2}\right)}+\frac{1}{\eta \omega}\right)^{2}}
\end{aligned}
$$

\section{Conclusions}

A general constitutive equation was derived to consider the interaction of the time- and cyclicdependent mechanical properties of viscoelastic materials, and solved for two specific loading patterns, 1) stress unloading from mean to zero stress level (recovery solution), and 2) load control sinusoidal loading (fatigue solution). To implement and validate these solutions, a loading pattern comprising a constant amplitude cyclic loading with repetitive interruptions was selected and applied to $\pm 45^{\circ}$ angleply glass/epoxy composite laminates. The evolution of the viscoelastic parameters was estimated at different percentages of fatigue life by adjusting the recovery solution to the experiments. In order to 
evaluate the accuracy of the constitutive equation implemented, the predictions of the fatigue solution were then compared with the experimental results in cyclic phases of the loading pattern. The following conclusions were drawn:

- As the number of cycles increased, the viscoelastic parameters decreased due to damage accumulation, except that corresponding to the remaining deformation that increased, as a result of the decreasing rate of the remaining strain.

- With the viscoelastic parameters calibrated, the predicted results of the fatigue solution could simulate the fatigue hysteresis loops, fatigue stiffness, and cyclic creep. All were in good agreement with the results of the experiments with the exception of the hysteresis loop area which was slightly underestimated at low stress levels.

- The fatigue solution enabled the average cyclic strain to be decomposed into the elastic, viscoelastic, and remaining strain components. As the number of cycles increased, all strain components increased as a result of fatigue damage formation and accumulation. It was observed that the elastic and viscoelastic strains increased gradually while the remaining strain increased more rapidly and was the main reason for the growth of the cyclic strain.

- The proposed constitutive equation was also able to predict the evolution of the storage modulus well while the predicted loss modulus and $\tan (\delta)$ were only slightly underestimated. It was observed that, as the number of cycles increased, the storage modulus decreased, while both the loss modulus and $\tan (\delta)$ increased.

- The proposed governing equation is applicable in cases where cyclic loading is interrupted for a sufficient length of time. Additionally, the constitutive equation is useable when there is an interaction between time- and cyclic-mechanical properties, and therefore it may not be used in fiber-dominated composites with negligible time-dependent mechanical properties.

\section{Acknowledgments}

The authors wish to acknowledge the support and funding of this research by the Swiss National Science Foundation (Grant No. 200021_156647/1).

\section{References:}

[1] Movahedi-Rad, A. V., Keller, T., \& Vassilopoulos, A. P. (2018). Interrupted tension-tension fatigue behavior of angle-ply GFRP composite laminates. International Journal of Fatigue, 113, 377-388. 
[2] Movahedi-Rad, A. V., Keller, T., \& Vassilopoulos, A. P. (2019). Creep effects on tension-tension fatigue behavior of angle-ply GFRP composite laminates. International Journal of Fatigue, 123, 144156.

[3] Tschoegl, N. W. (1998). Time-dependence in materials. In Progress and Trends in Rheology V (pp. 1-3). Steinkopff, Heidelberg.

[4] Mallick, P. K., \& Zhou, Y. (2004). Effect of mean stress on the stress-controlled fatigue of a short Eglass fiber reinforced polyamide-6, 6. International journal of fatigue, 26(9), 941-946.

[5] Haward, R. N., \& Thackray, G. 1. (1968). The use of a mathematical model to describe isothermal stress-strain curves in glassy thermoplastics. Proc. R. Soc. Lond. A, 302(1471), 453-472.

[6] Boyce, M. C., Parks, D. M., \& Argon, A. S. (1988). Large inelastic deformation of glassy polymers. Part I: rate dependent constitutive model. Mechanics of Materials, 7(1), 15-33.

[7] Hizoum, K., Rémond, Y., Bahlouli, N., Oshmyan, V., Patlazhan, S., \& Ahzi, S. (2006). Non linear strain rate dependency and unloading behavior of semi-crystalline polymers. Oil \& Gas Science and Technology-Revue de l'IFP, 61(6), 743-749.

[8] Yakimets, I., Lai, D., \& Guigon, M. (2007). Model to predict the viscoelastic response of a semicrystalline polymer under complex cyclic mechanical loading and unloading conditions. Mechanics of Time-Dependent Materials, 11(1), 47-60.

[9] Drozdov, A. D. (2009). Constitutive model for cyclic deformation of perfluoroelastomers. Mechanics of Time-Dependent Materials, 13(3), 275-299.

[10] Ayoub, G., Zaïri, F., Naït-Abdelaziz, M., \& Gloaguen, J. M. (2010). Modelling large deformation behaviour under loading-unloading of semicrystalline polymers: application to a high density polyethylene. International Journal of Plasticity, 26(3), 329-347.

[11] Vandenbroucke, A., Laurent, H., Hocine, N. A., \& Rio, G. (2010). A Hyperelasto-Visco-Hysteresis model for an elastomeric behaviour: experimental and numerical investigations. Computational Materials Science, 48(3), 495-503.

[12] Drozdov, A. D. (2012). Cyclic viscoelastoplasticity of polypropylene/nanoclay hybrids. Computational Materials Science, 53(1), 396-408.

[13] Colak, O. U. (2005). Modeling deformation behavior of polymers with viscoplasticity theory based on overstress. International Journal of Plasticity, 21(1), 145-160.

[14] Krasnobrizha, A., Rozycki, P., Cosson, P., \& Gornet, L. (2015, July). Modelling the hysteresis composite behavior using an elasto-plasto-damage model with fractional derivatives. In 20th International Conference on Composite Materials-ICCM20.

[15] Schapery, R. A. (1984). Correspondence principles and a generalizedJ integral for large deformation and fracture analysis of viscoelastic media. International journal of fracture, 25(3), 195223.

[16] Zhang, C., \& Moore, I. D. (1997). Nonlinear mechanical response of high density polyethylene. Part II: Uniaxial constitutive modeling. Polymer Engineering \& Science, 37(2), 414-420.

[17] Khan, A., \& Zhang, H. (2001). Finite deformation of a polymer: experiments and modeling. International Journal of Plasticity, 17(9), 1167-1188. 
[18] Fiugge, W., Viscoelasticity. Blaisdell, Waltham, MA, 1964.

[19] Ferry, J. D., Viscoelastic Properties of Polymers, 3rd edn. John Wiley and Sons, New York, 1980.

[20] Guedes, Rui Miranda, ed. Creep and fatigue in polymer matrix composites. Elsevier, 2010.

[21] Schapery, R. A. (1969). On the characterization of nonlinear viscoelastic materials. Polymer Engineering \& Science, 9(4), 295-310.

[22] Zaoutsos, S. P., Papanicolaou, G. C., \& Cardon, A. H. (1998). On the non-linear viscoelastic behaviour of polymer-matrix composites. Composites Science and Technology, 58(6), 883-889.

[23] Whitworth, H. A. (1997). A stiffness degradation model for composite laminates under fatigue loading. Composite structures, 40(2), 95-101.

[24] Machado, R. D., Abdalla Filho, J. E., \& da Silva, M. P. (2008). Stiffness loss of laminated composite plates with distributed damage by the modified local Green's function method. Composite Structures, 84(3), 220-227.

[25] Wu, F., \& Yao, W. (2010). A fatigue damage model of composite materials. International Journal of Fatigue, 32(1), 134-138.

[26] Zhang, Y., Vassilopoulos, A. P., \& Keller, T. (2010). Fracture of adhesively-bonded pultruded GFRP joints under constant amplitude fatigue loading. International Journal of Fatigue, 32(7), 979-987.

[27] Vassilopoulos AP, Keller T. Fatigue of fiber-reinforced composites. Springer Science \& Business Media, 2011.

[28] Sarfaraz, R., Vassilopoulos, A. P., \& Keller, T. (2011). Experimental investigation of the fatigue behavior of adhesively-bonded pultruded GFRP joints under different load ratios. International Journal of Fatigue, 33(11), 1451-1460.

[29] Shahverdi, M., Vassilopoulos, A. P., \& Keller, T. (2012). A total fatigue life model for the prediction of the R-ratio effects on fatigue crack growth of adhesively-bonded pultruded GFRP DCB joints. Composites Part A: Applied Science and Manufacturing, 43(10), 1783-1790.

[30] Kennedy, C. R., Brádaigh, C. M. Ó., \& Leen, S. B. (2013). A multiaxial fatigue damage model for fibre reinforced polymer composites. Composite Structures, 106, 201-210.

[31] Mohammadi, B., Fazlali, B., \& Salimi-Majd, D. (2017). Development of a continuum damage model for fatigue life prediction of laminated composites. Composites Part A: Applied Science and Manufacturing, 93, 163-176.

[32] Miyano, Y., Nakada, M., McMurray, M. K., \& Muki, R. (1997). Prediction of flexural fatigue strength of CRFP composites under arbitrary frequency, stress ratio and temperature. Journal of Composite Materials, 31(6), 619-638.

[33] Petermann, J., \& Schulte, K. (2002). The effects of creep and fatigue stress ratio on the long-term behaviour of angle-ply CFRP. Composite Structures, 57(1-4), 205-210.

[34] Vieille, B., Albouy, W., \& Taleb, L. (2014). About the creep-fatigue interaction on the fatigue behaviour of off-axis woven-ply thermoplastic laminates at temperatures higher than Tg. Composites Part B: Engineering, 58, 478-486. 
[35] Sayyidmousavi, A., Bougherara, H., \& Fawaz, Z. (2015). The role of viscoelasticity on the fatigue of angle-ply polymer matrix composites at high and room temperatures-a micromechanical approach. Applied Composite Materials, 22(3), 307-321.

[36] Movahedi-Rad, A. V., Keller, T., \& Vassilopoulos, A. P. (2018). Fatigue damage in angle-ply GFRP laminates under tension-tension fatigue. International Journal of Fatigue, 109, 60-69.

[37] Savvilotidou, M., Keller, T., \& Vassilopoulos, A. P. (2017). Fatigue performance of a cold-curing structural epoxy adhesive subjected to moist environments. International Journal of Fatigue, 103, 405414.

[38] Sun, C. T., \& Chim, E. S. (1981). Fatigue retardation due to creep in a fibrous composite. In Fatigue of Fibrous Composite Materials. ASTM International.

[39] ASTM, D3171-99. Standard test methods for constituent content of composite materials. ASTM International; 2002.

[40] ASTM D3039/D3039M-14 Standard Test Method for Tensile Properties of Polymer Matrix Composite Materials. ASTM International; 2014.

[41] ASTM, D7791-17. Standard Test Method for Uniaxial Fatigue Properties of Plastics. ASTM International; 2017.

[42] Krause O, Philippidis TP. General test specification Optimat report: OB_TC_R015 available on line from $<$ https://www.wmc.eu/public_docs/10157_005.pdf $>$.

[43] Kawai, M., \& Masuko, Y. (2004). Creep behavior of unidirectional and angle-ply T800H/3631 laminates at high temperature and simulations using a phenomenological viscoplasticity model. Composites Science and Technology, 64(15), 2373-2384.

[44] Ascione, L., Berardi, V. P., \& D'Aponte, A. (2011). A viscoelastic constitutive law for FRP materials. International Journal for Computational Methods in Engineering Science and Mechanics, 12(5), 225-232.

[45] Xu, Y. (2009). Creep behavior of natural fiber reinforced polymer composites.

[46] Boggs, P. T., \& Rogers, J. E. (1990). Orthogonal distance regression. Contemporary Mathematics, 112, 183-194.

[47] Chawla, K. K., \& Meyers, M. A. (1999). Mechanical behavior of materials (p. 217). Upper Saddle River: Prentice Hall.

[48] Brinson, H. F., \& Brinson, L. C. (2008). Polymer engineering science and viscoelasticity. New York: Springer, 66, 79. 


\section{Figures:}

Fig. 1. Basis of constitutive equation to describe behavior of viscoelastic materials

Fig. 2. Flowchart of proposed modeling method.

Fig. 3. Schematic representation of loading pattern in interrupted fatigue experiments.

Fig. 4. Elastic, three-stage viscoelastic recovery, and remaining strains of specimen Intf-0.1-58-a after third loading block.

Fig. 5. Experimental and modeled recovery curves for different loading blocks and stress levels, (a) Intf-0.1-58-a, and (b) Intf-0.1-49-a.

Fig. 6. Variation of viscoelastic parameters (a) $E$, (b) $E_{1}$, (c) $\eta_{1}$, (d) $E_{2}$, (e) $\eta_{2}$, (f) $E_{3}$, (g) $\eta_{3}$, and (h) $\eta$ versus normalized number of cycles at different stress levels.

Fig. 7. Applied sinusoidal stress and strain response in viscoelastic material.

Fig. 8. Experimental (dots) and predicted fatigue (solid line) hysteresis loops at different number of cycles (N) at two stress levels, (a) Intf-0.1-64-b, and (b) Intf-0.1-49-a.

Fig. 9. Hysteresis loop area versus normalized number of cycles at different stress levels (a) Intf-0.1-64-b, and (b) Intf-0.1-49-a.

Fig. 10. Fatigue stiffness versus normalized number of cycles at different stress levels (a) Intf-0.1-64-b, and (b) Intf-0.1-49-a.

Fig. 11. Evolution of average (a) cyclic, (b) elastic, (c) viscoelastic, and (d) remaining strain at different stress levels.

Fig. 12. Variation of storage modulus, loss modulus, and $\tan (\delta)$ during fatigue loading at different stress levels (a) Intf-0.1-64-b, and (b) Intf-0.1-49-a. 


$$
\begin{gathered}
\varepsilon(t)=\frac{\sigma(t)}{E} \\
\varepsilon(t)=\frac{\sigma(t)}{E_{1}+\eta_{1} \frac{\partial}{\partial t}} \\
\varepsilon(t)=\frac{\sigma(t)}{E_{2}+\eta_{2} \frac{\partial}{\partial t}} \\
\varepsilon(t)=\frac{\sigma(t)}{E_{i}+\eta_{i} \frac{\partial}{\partial t}} \\
\varepsilon(t)=\frac{\sigma(t)}{\eta \frac{\partial}{\partial t}}
\end{gathered}
$$

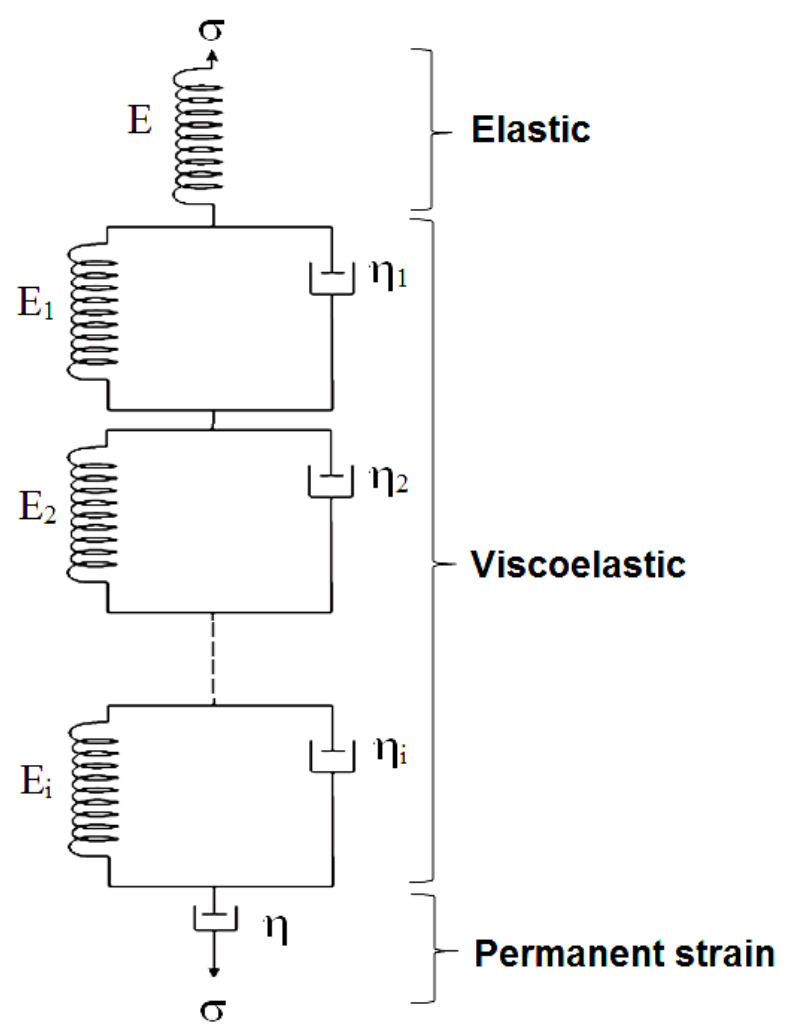

Fig. 1. Basis of constitutive equation to describe behavior of viscoelastic materials

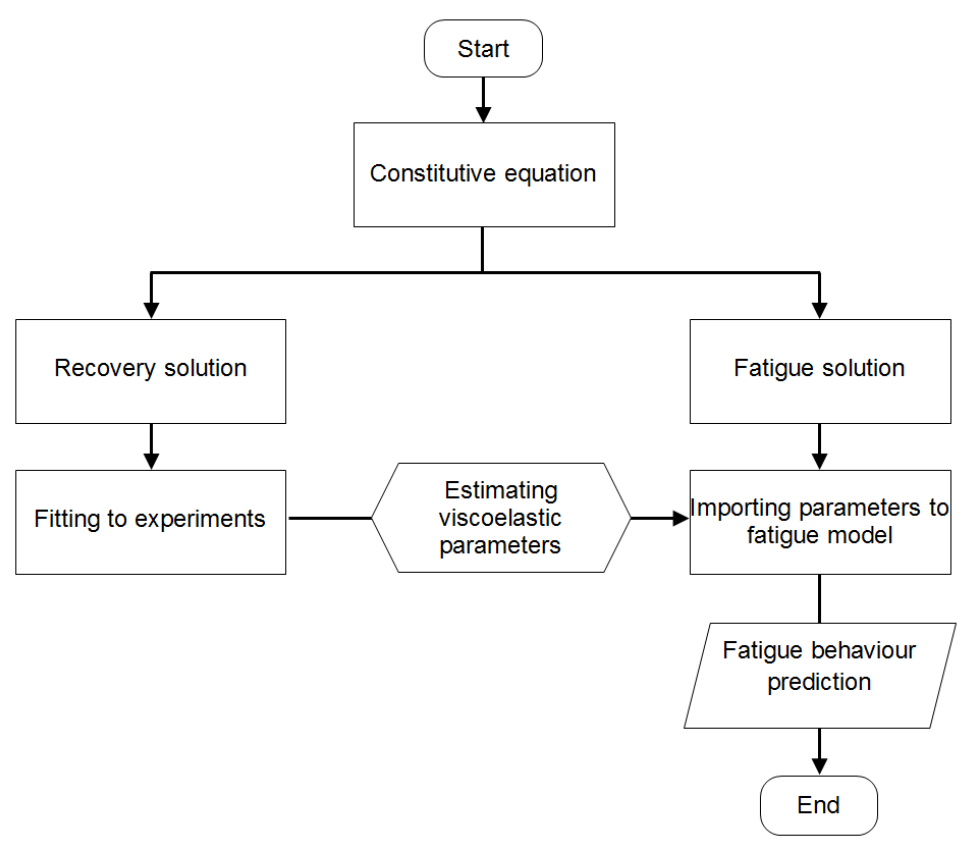

Fig. 2. Flowchart of implementation of constitutive equation. 


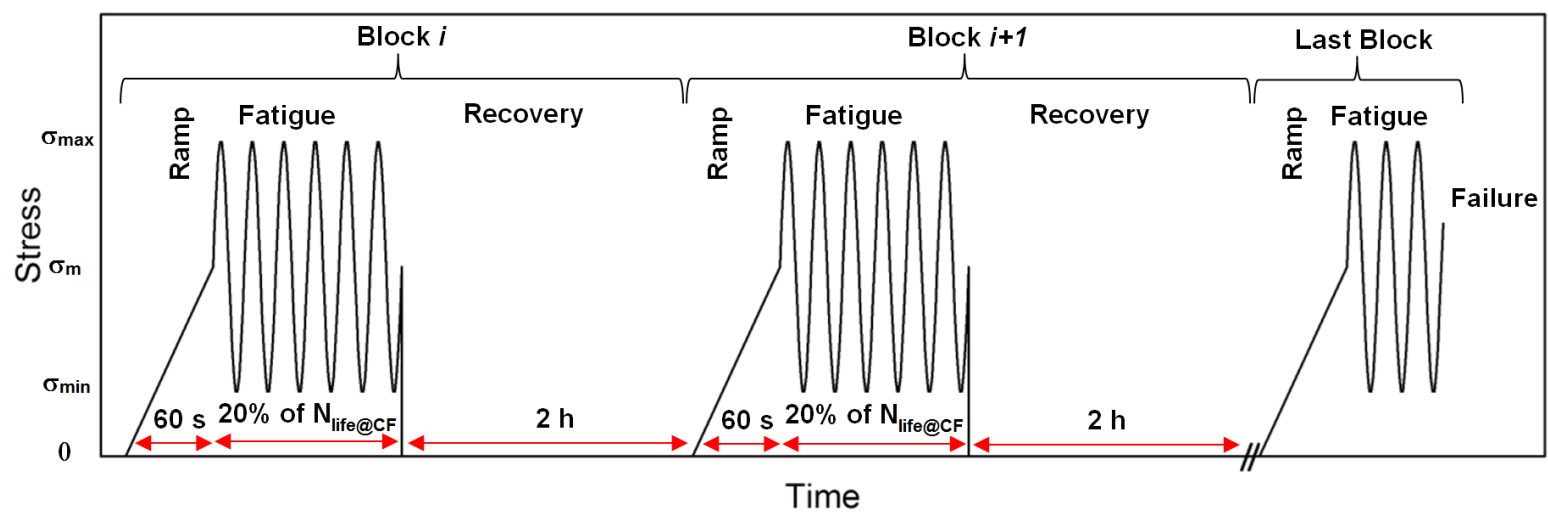

Fig. 3. Schematic representation of loading pattern in interrupted fatigue experiments.

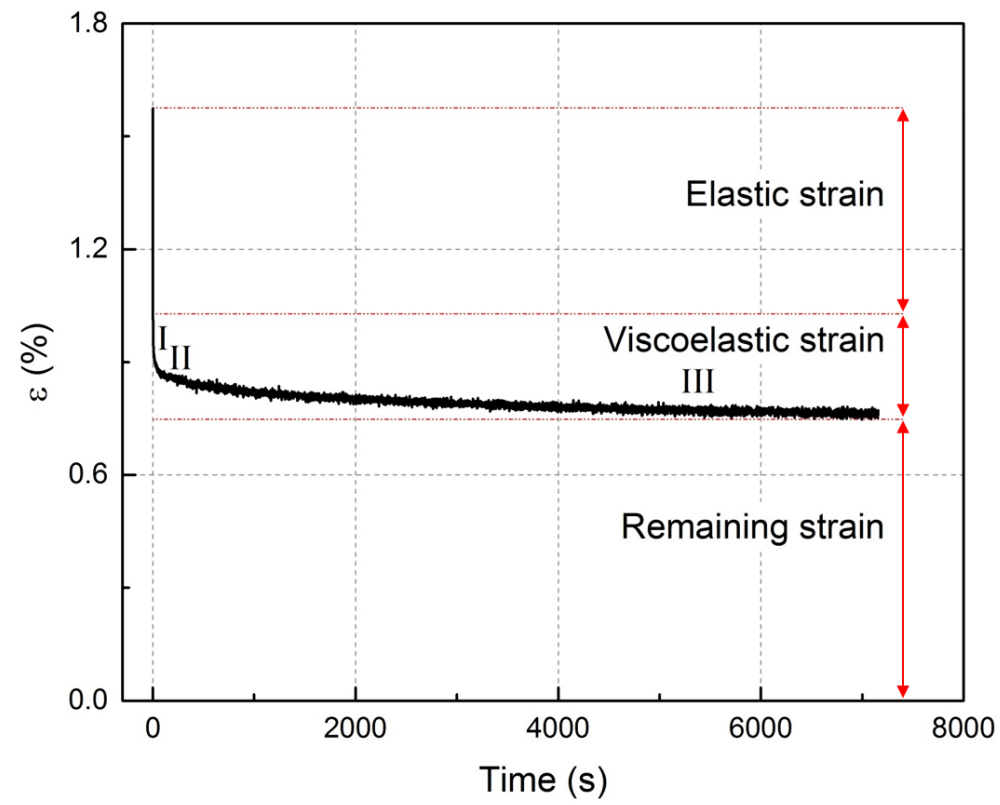

Fig. 4. Elastic, three-stage viscoelastic recovery, and remaining strains of specimen Intf-0.1-58-a after third loading block. 

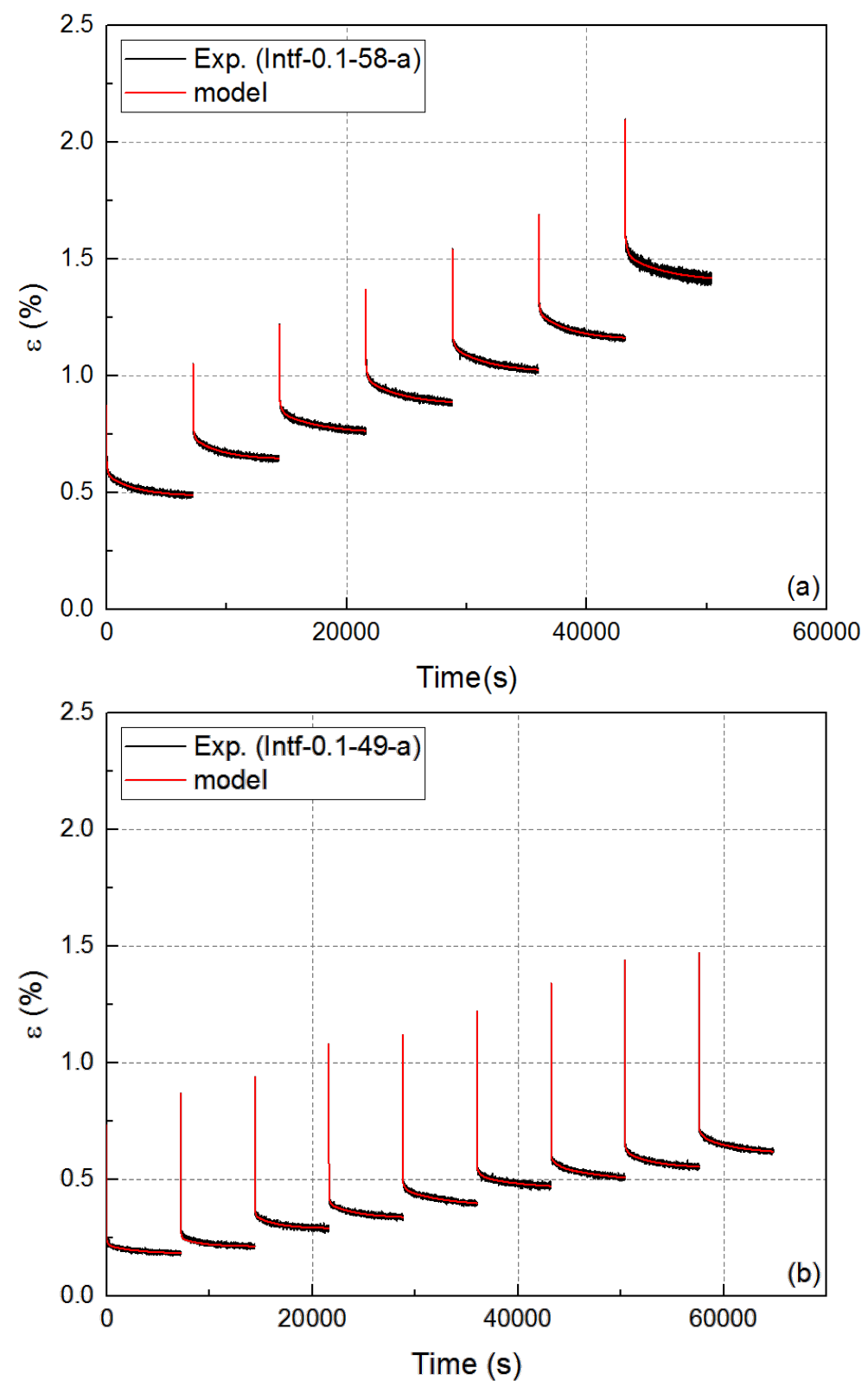

Fig. 5. Experimental and modeled recovery curves for different loading blocks and stress levels, (a) Intf-0.1-58-a, and (b) Intf-0.1-49-a. 

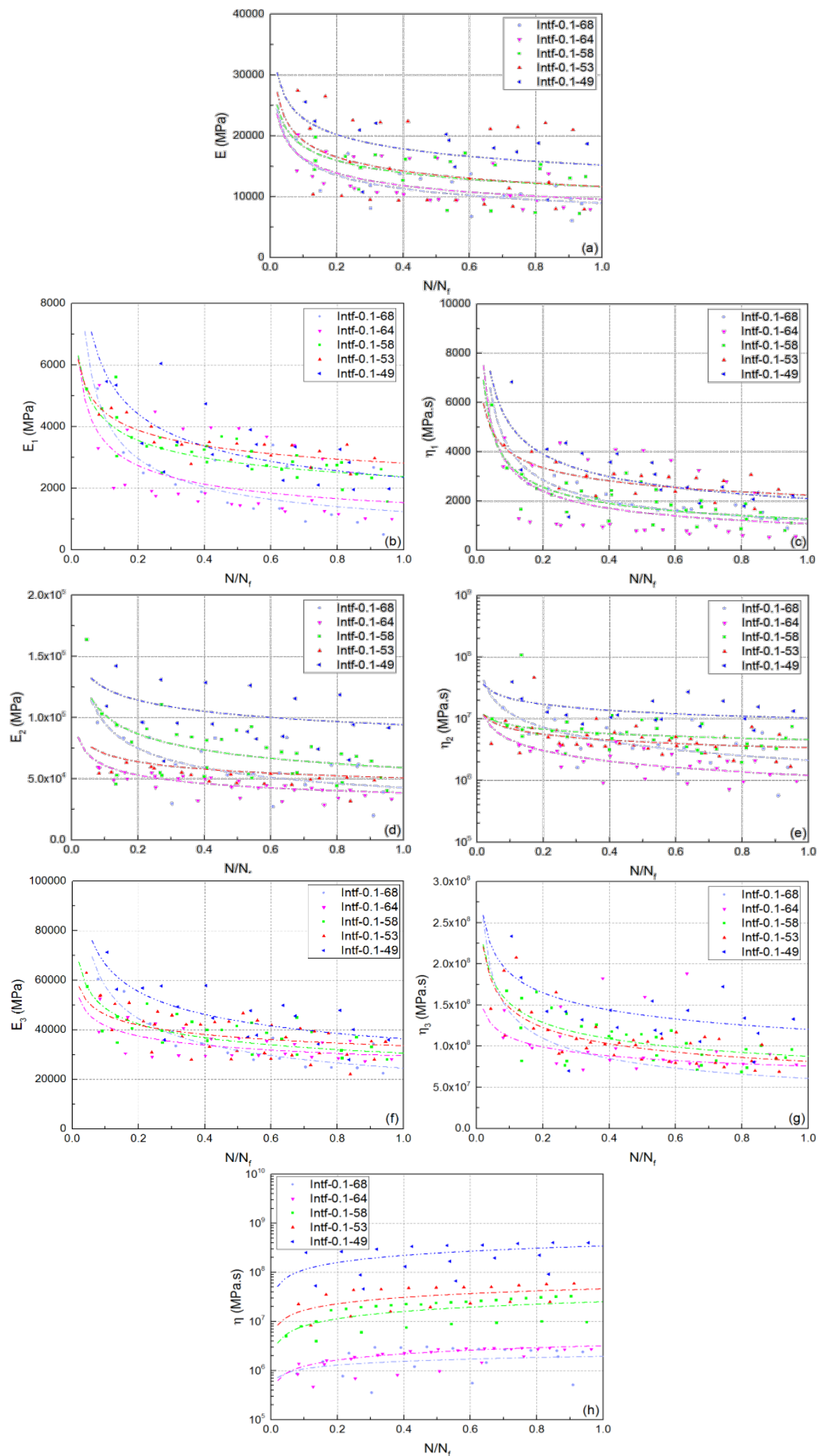

Fig. 6. Variation of viscoelastic parameters (a) $E$, (b) $E_{1}$, (c) $\eta_{1}$, (d) $E_{2}$, (e) $\eta_{2}$, (f) $E_{3}$, (g) $\eta_{3}$, and (h) $\eta$ versus normalized number of cycles at different stress levels. 


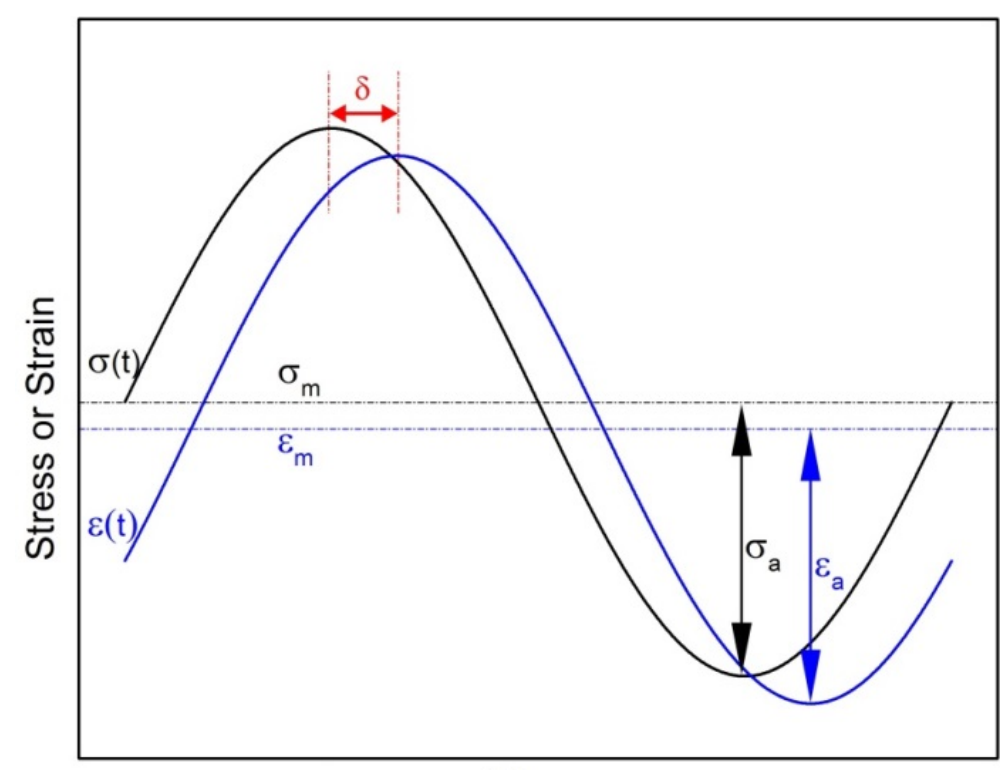

Time

Fig. 7. Applied sinusoidal stress and strain response in viscoelastic material. 

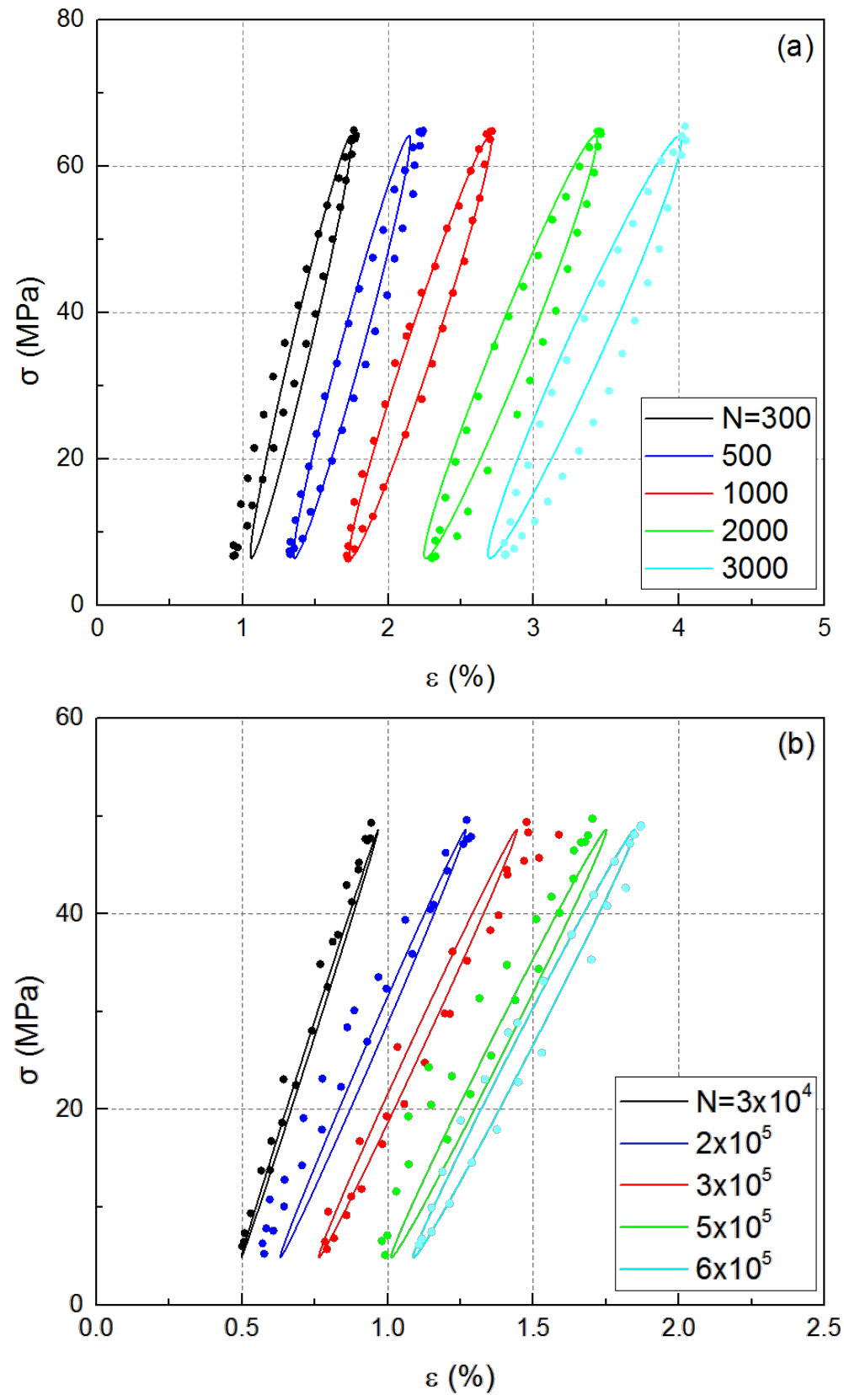

Fig. 8. Experimental (dots) and predicted fatigue (solid line) hysteresis loops at different number of cycles $(\mathrm{N})$ at two stress levels, (a) Intf-0.1-64-b, and (b) Intf-0.1-49-a. 

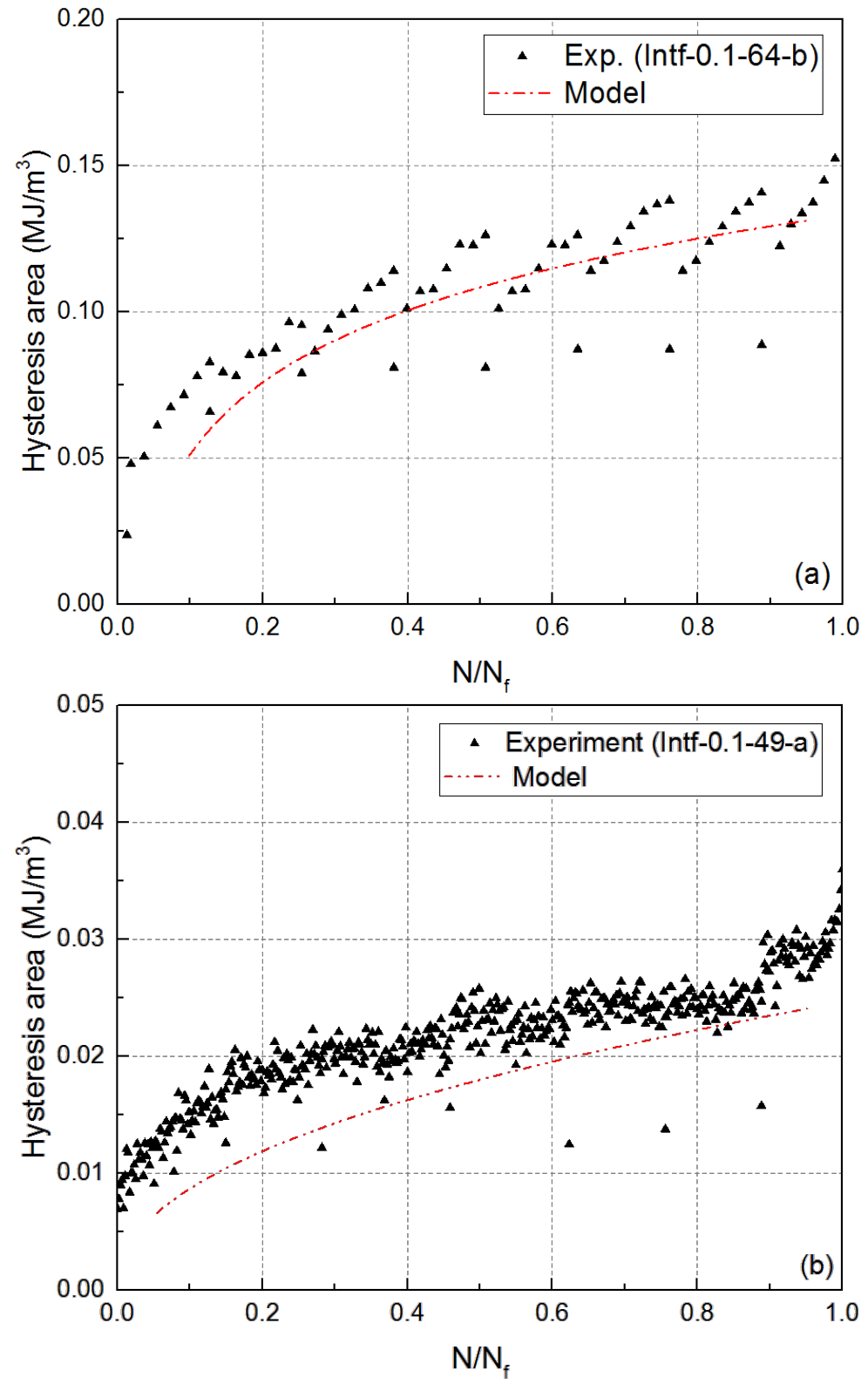

Fig. 9. Hysteresis loop area versus normalized number of cycles at different stress levels (a) Intf-0.1-64-b, and (b) Intf-0.1-49-a. 

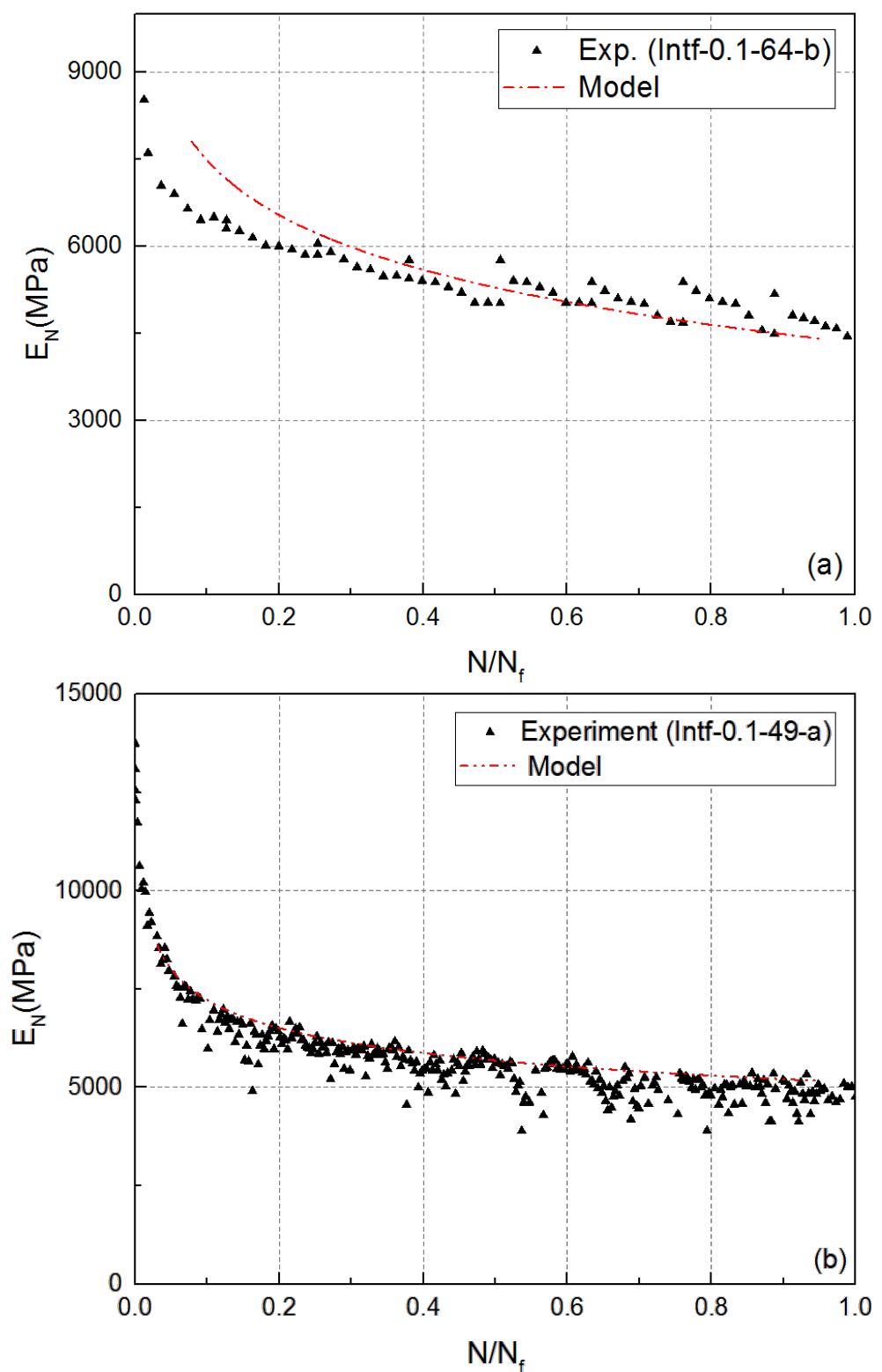

Fig. 10. Fatigue stiffness versus normalized number of cycles at different stress levels (a) Intf-0.1-64-b, and (b) Intf-0.1-49-a. 

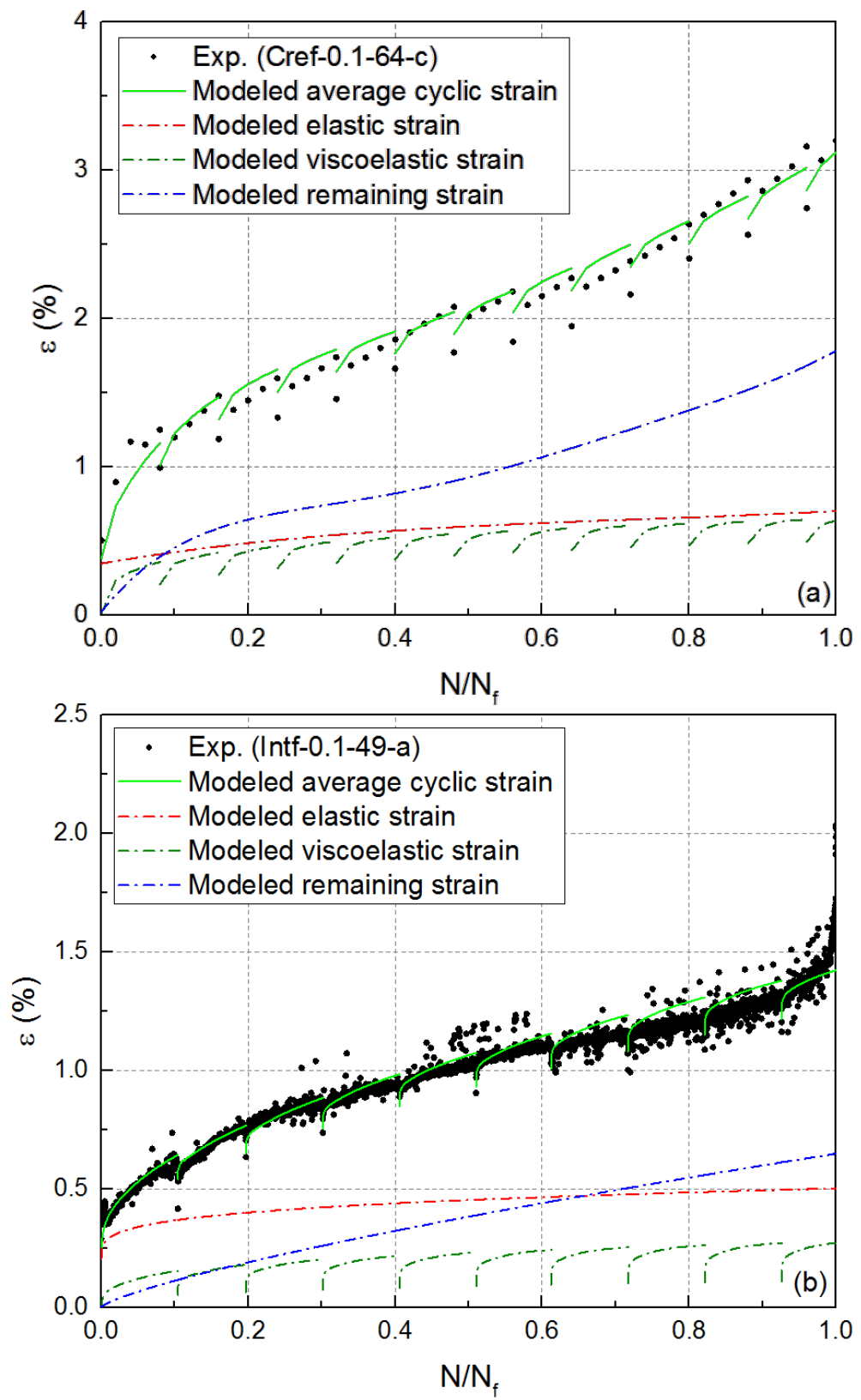

Fig. 11. Evolution of average cyclic, elastic, viscoelastic, and remaining strain at different stress levels (a) Intf-0.164-c, and (b) Intf-0.1-49-a. 

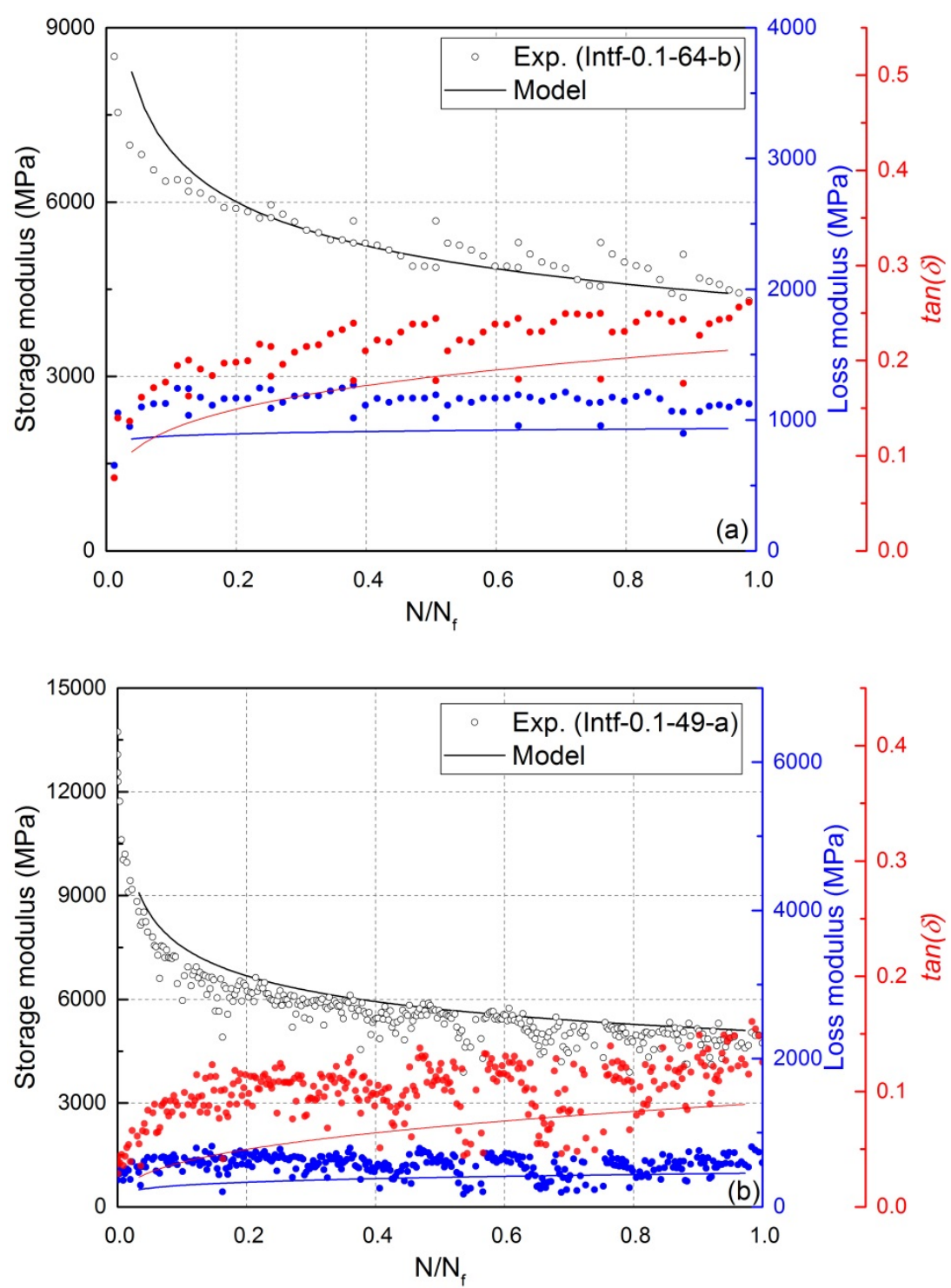

Fig. 12. Variation of storage modulus, loss modulus, and $\tan (\delta)$ during fatigue loading at different stress levels (a) Intf-0.1-64-b, and (b) Intf-0.1-49-a. 


\section{Annex I:}

The applied loading pattern in sinusoidal cyclic loading is;

$\sigma(t)=\sigma_{m}+\sigma_{a} \sin (\omega t)$

The response of Maxwell and Kelvin units to this loading pattern is as follows:

\section{Solution for one Maxwell unit:}

The constitutive equation for a Maxwell unit consisting of a series of a spring and a dashpot is:

$\sigma(t)+\frac{\eta}{E} \dot{\sigma}(t)=\eta \dot{\varepsilon}(t)$

By inserting Eq. I-1 into Eq. I-2 and rearranging, the following equation is obtained:

$\varepsilon(t)=\frac{\sigma_{m}}{\eta} t+\frac{\sigma_{a}}{\eta \omega} \cos (\omega t)-\frac{\sigma_{a}}{E} \sin (\omega t)+C_{1}$

\section{Solution for one Kelvin unit:}

The constitutive equation for a Kelvin unit consisting of a parallel pair of a spring and a dashpot is:

$\sigma(t)=E \varepsilon(t)+\eta \dot{\varepsilon}(t)$

By inserting Eq. I-1 into Eq. I-4 and rearranging, the obtained equation is:

$\dot{\varepsilon}(t)+\lambda \varepsilon(t)=\frac{\sigma_{m}}{\eta}+\frac{\sigma_{a}}{\eta} \sin (\omega t)$

By multiplying the integral factor of $e^{\lambda t}$ to both sides of Eq. I-5, rearranging, and integrating the resulting equation, the following relationship is obtained:

$\varepsilon(t) e^{\lambda t}=\frac{\sigma_{m}}{\eta \lambda} e^{\lambda t}+\frac{\sigma_{a}}{\eta} \int e^{\lambda t} \sin (\omega t) d t$

By using the theorem of partial integration twice, the following relationship results:

$\varepsilon(t)=\frac{\sigma_{m}}{E}+C_{2} \frac{\sigma_{m}}{\eta} e^{\lambda t}+\left(\frac{\sigma_{a}}{\eta_{i}} \frac{\lambda}{\lambda^{2}+\omega^{2}} \sin (\omega t)-\frac{\sigma_{a}}{\eta} \frac{\omega}{\lambda^{2}+\omega^{2}} \cos (\omega t)\right)$

\section{Solution for one Maxwell and three Kelvin units:}

The general solution for one Maxwell and three Kelvin units is the summation of Eq. I-3 and Eq. I-7 as follows: 


$$
\begin{aligned}
& \varepsilon(t)=\frac{\sigma_{m}}{E}+\sum_{i=1}^{i=3} \frac{\sigma_{m}}{E_{i}}\left(1-e^{-\frac{E_{i}}{\eta_{i}}\left(t-t_{0}\right)}\right)+\frac{\sigma_{m}}{\eta}\left(t-t_{0}\right)+\sum_{i=1}^{i=3}\left(\frac{\sigma_{a}}{\eta_{i}} \frac{\lambda_{i}}{\lambda_{i}^{2}+\omega^{2}}+\frac{\sigma_{a}}{E}\right) \sin (\omega t)- \\
& \left(\frac{\sigma_{a}}{\eta_{i}} \frac{\omega}{\lambda_{i}^{2}+\omega^{2}}+\frac{\sigma_{a}}{\eta \omega}\right) \cos (\omega t)
\end{aligned}
$$

which can also be written as:

$$
\begin{aligned}
& \varepsilon(t)=\frac{\sigma_{m}}{E}+\sum_{i=1}^{i=3} \frac{\sigma_{m}}{E_{i}}\left(1-e^{-\frac{E_{i}}{\eta_{i}}\left(t-t_{0}\right)}\right)+\frac{\sigma_{m}}{\eta}\left(t-t_{0}\right)+ \\
& \sigma_{a} \sqrt{\left(\sum_{i=1}^{i=3} \frac{1}{\eta_{i}} \frac{\lambda}{\lambda_{i}^{2}+\omega^{2}}+\frac{1}{E}\right)^{2}+\left(\sum_{i=1}^{i=3} \frac{1}{\eta_{i}} \frac{\omega}{\lambda_{i}^{2}+\omega^{2}}+\frac{1}{\eta \omega}\right)^{2} \sin \left(\omega t+\tan ^{-1}\left(\frac{\sum_{i=1}^{i=3} \frac{\omega}{\eta_{i}\left(\lambda_{i}^{2}+\omega^{2}\right)}+\frac{1}{\eta \omega}}{\sum_{i=1}^{i=3} \frac{\lambda}{\eta_{i}\left(\lambda_{i}^{2}+\omega^{2}\right)}+\frac{1}{E}}\right)\right)}
\end{aligned}
$$

The previous equation can be simplified to the following form:

$\varepsilon(t)=\varepsilon_{c}(t)+\varepsilon_{a} \sin (\omega t-\delta)$

where

$$
\begin{aligned}
& \varepsilon_{c}(t)=\frac{\sigma_{m}}{E}+\sum_{i=1}^{i=3} \frac{\sigma_{m}}{E_{i}}\left(1-e^{-\lambda_{i}\left(t-t_{0}\right)}\right)+\frac{\sigma_{m}}{\eta}\left(t-t_{0}\right) \\
& \varepsilon_{a}=\sigma_{a} \sqrt{\left(\sum_{i=1}^{i=3} \frac{1}{\eta_{i}} \frac{\lambda_{i}}{\lambda_{i}^{2}+\omega^{2}}+\frac{1}{E}\right)^{2}+\left(\sum_{i=1}^{i=3} \frac{1}{\eta_{i}} \frac{\omega}{\lambda_{i}^{2}+\omega^{2}}+\frac{1}{\eta \omega}\right)^{2}} \\
& \delta=\tan ^{-1}\left(\frac{\sum_{i=1}^{i=3} \frac{\omega}{\eta_{i}\left(\lambda_{i}^{2}+\omega^{2}\right)}+\frac{1}{\eta \omega}}{\sum_{i=3}^{i=3} \frac{\lambda_{i}}{\eta_{i}\left(\lambda_{i}^{2}+\omega^{2}\right)}+\frac{1}{E}}\right)
\end{aligned}
$$

\section{Annex II:}

The evolution of the storage and loss moduli during the fatigue loading can be calculated as follows. By considering:

$$
\begin{aligned}
& b=\sum_{i=1}^{i=3} \frac{1}{\eta_{i}} \frac{\omega}{\lambda_{i}^{2}+\omega^{2}}+\frac{1}{\eta \omega} \\
& a=\sum_{i=1}^{i=3} \frac{1}{\eta_{i}} \frac{\lambda}{\lambda_{i}^{2}+\omega^{2}}+\frac{1}{E}
\end{aligned}
$$

the following equations are valid:

$$
E^{*}=\sqrt{{E^{\prime}}^{2}+E^{\prime \prime 2}}=\frac{1}{\sqrt{a^{2}+b^{2}}}
$$


$\tan (\delta)=\frac{E^{\prime \prime}}{E^{\prime}}=\frac{b}{a}$

By considering both Eq. II-3 and Eq. II-4, the magnitude of storage and loss moduli were calculated according to Eq. II-5 and Eq. II-6;

$$
\begin{aligned}
& E^{\prime}=\frac{a}{a^{2}+b^{2}} \\
& E^{\prime \prime}=\frac{b}{a^{2}+b^{2}}
\end{aligned}
$$

